\title{
Carbon Monoxide and Iron Modulate Plasmatic Coagulation in Alzheimer's disease
}

\author{
Vance G. Nielsen ${ }^{1, *}$, Etheresia Pretorius ${ }^{2}$, Janette Bester ${ }^{2}$, Wayne K. Jacobsen ${ }^{1}$, Patrick K. Boyle ${ }^{1}$, \\ Joao P. Reinhard ${ }^{1}$
}

${ }^{I}$ The Department of Anesthesiology, the University of Arizona College of Medicine, Tucson, Arizona, USA;
and the Department of Physiology; ${ }^{2}$ Faculty of Health Sciences, University of Pretoria, Arcadia, South
Africa

Abstract: Alzheimer's disease (AD) is a significant source of morbidity and mortality for millions of people worldwide, and multiple potential etiologies have been postulated to contribute to AD. Among these, spontaneous cerebral emboli and increased cerebral and circulating heme oxygenase (Hmox) activity in AD patients are of particular interest, as two of the products of Hmox activity, carbon monoxide (CO) and iron enhance plasmatic coagulation and modify the ultrastructure of thrombi. We hypothesized that patients afflicted with $\mathrm{AD}$ would have coagulation kinetics modulated by $\mathrm{CO}$ and iron. Using

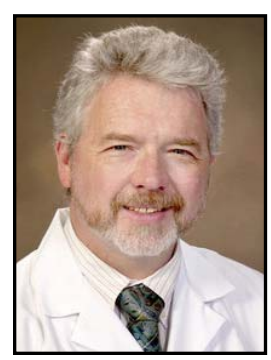
viscoelastic assessments of coagulation, it was determined with a small cohort $(n=11)$ of AD patients that all had enhancement of coagulation by $\mathrm{CO}$, iron, or both. In a complementary fashion, it was determined that a separate cohort $(n=12)$ of $\mathrm{AD}$ patients had thrombi with ultrastructural features consistent with iron and $\mathrm{CO}$ exposure as assessed with scanning electron microscopy. Further, when stratified by normal or abnormally increased serum ferritin concentrations (which can be increased by Hmox), the AD patients with abnormal ferritin concentrations had significantly thinner fibrin fiber diameters, not unlike that noted when normal plasma is mixed with iron or CO. In sum, AD patients were noted to have plasmatic coagulation kinetic and thrombus ultrastructural changes consistent with exposure to $\mathrm{CO}$ and iron. Future investigation of $\mathrm{CO}$ and iron in the pathogenesis of Alzheimer's disease is warranted.

Keywords: Alzheimer's disease, carbon monoxide, iron, heme oxygenase, scanning electron microscopy, thrombelastography.

\section{INTRODUCTION}

Alzheimer's disease (AD) is a major source of morbidity and mortality for millions of people worldwide, and intense investigation has led to multiple etiological constructs contributing to $\mathrm{AD}$ [1-12]. In addition to the recently reviewed, original 'amyloid cascade hypothesis' [1], the development and progression of $\mathrm{AD}$ have been associated with viral infection [2], vascular damage from pulse pressure [3], regional deposition of metals, such as iron, resulting in oxidant injury [4-8], metabolic syndrome and morbid obesity [8-10], and long term exposure to spontaneous cerebral microemboli $[11,12]$. Of these various paradigms, the notion that spontaneous emboli, most likely due to systemic hypercoagulability, is of particular interest. AD patients have been documented to have abnormally increased activated Factor VII, von Willebrand factor and prothrombin $1+2$ fragment in their circulation [13], the substrate and result of intravascular thrombin generation. Of interest, while patients with $\mathrm{AD}$ were noted to have fibrinogen concentrations that on average were not abnormally increased [13], another

*Address correspondence to this author at the Department of Anesthesiology, the University of Arizona College of Medicine, P.O. Box 245114; 1501 North Campbell Avenue, Tucson, AZ 85724-5114, USA; Tel: (520) 626-7195; Fax: (520) 626-6943;

E-mail:vgnielsen@email.arizona.edu work noted that patient with mild cognitive impairment were at greater risk of more rapid advancement to $\mathrm{AD}$ if their circulating fibrinogen concentration was $>300 \mathrm{mg} / \mathrm{dl}$ [14]. Further, patients with AD have been noted to have plasma thrombus fibrin polymerization that is structurally similar to iron-exposed plasma [15], and AD patients with abnormally increased ferritin concentrations showed red blood cell morphological changes, with increased membrane stiffness documented with atomic force microscopy [16]. In sum, there is biochemical and ultrastructural evidence of circulating abnormal intravascular thrombin generation, rigid red blood cells, and consequent abnormally appearing fibrin polymerization in thrombi obtained from patients with $\mathrm{AD}$, all of which could contribute to spontaneous emboli.

Given the aforementioned morphological changes in fibrin in plasma obtained from $\mathrm{AD}$ patients, it is of particular interest that carbon monoxide $[\mathrm{CO}]$ and iron enhance plasmatic coagulation kinetics and modify ultrastructure by modulating fibrinogen [17]. Iron decreases the onset time of coagulation and enhances the speed of clot formation, $\mathrm{CO}$ enhances the speed of clot formation and final clot strength, and iron and $\mathrm{CO}$ interact kinetically in an additive fashion [17]. Further, both iron and $\mathrm{CO}$ act as antifibrinolytic agents when tissue-type plasminogen activator (tPA) is the fibrinolysin [17]. These facts are of importance in the setting of $\mathrm{AD}$, as the endogenous enzyme system responsible for heme catabolism, heme oxygenase (Hmox), has been found to be enhanced in both the brain and circulation of $\mathrm{AD}$ 
patients [18-21], and Hmox catabolism of heme results in the release of iron and $\mathrm{CO}$ [22]. In contrast to the aforementioned data, in a recent review [23] $\mathrm{CO}$ has been noted to act as an anti-apoptotic agent that decreases the generation of reactive oxygen species, especially in situations of acute neurological ischemia-reperfusion injury in rodent models. However, inhibition of Hmox in a murine model of AD involving a double transgenic mouse $\left(\mathrm{APP}_{\text {swe }} / \mathrm{PS}_{\triangle \mathrm{Eg}}\right)$ significantly diminished the behavioral deficits and neuropathological changes over time compared to mice not administered a Hmox inhibitor [24]. When all these data are considered as a whole, Hmox enhanced coagulation could play a role in spontaneous thrombus formation not just in the peripheral circulation as documented by previous ultrasonic investigations [11,12], but perhaps more regionally (and intensely) in the microcirculation of the brain itself.

The first goal of this study was to determine if iron and $\mathrm{CO}$ enhanced coagulation/diminished fibrinolysis was present in plasma obtained from $\mathrm{AD}$ patients using viscoelastic assessments of coagulation and fibrinolytic kinetics as previously described [25-27]. The second goal was to image thrombi obtained from patients with AD with various degrees of iron exposure/inflammation with scanning electron microscopy (SEM), and examine these clots for signs of iron and $\mathrm{CO}$ modified fibrin polymer formation [17].

\section{MATERIALS AND METHODS}

\section{Viscoelastic Plasma Coagulation and Fibrinolysis Analyses.}

All viscoelastic method-based experimentation was completed at the University of Arizona. To establish normal laboratory ranges for the subsequently described assays, human plasma (George King Bio-Medical, Overland Park, KS, USA) anticoagulated with sodium citrate was obtained, which was composed of plasma from 30 individuals (15 males, 15 females; mean age 30 years, with range of 20 years to 47 years). All subjects were confirmed to be disease-free, nonsmokers, and not pregnant.

With regard to $\mathrm{AD}$ patient samples, they were obtained from a commercial vendor (PrecisionMed, Inc., Solana Beach, CA, USA). This vendor collects various body fluids (e.g., cerebral spinal fluid, blood) from patients being monitored over time that are diagnosed with minimal cognitive impairment (MCI) or Alzheimer's disease. The patient centers involved are located throughout the United States, with ethical oversight provided by the Western Institutional Review Board (Puyallup, WA, USA). The protocol number for this particular study was \#8009. The AD patients had to meet the following criteria for inclusion: mini mental state exam (MMSE) score $\geq 14$ to $\leq 28$; sign an approved written consent; agree to venipuncture; be $\geq 50$ years of age; have a brain magnetic resonance imaging or computed tomographic study excluding other causes of neurological compromise; Hachinski score $\leq 4$; have a diagnosis of dementia or MCI established by clinical exam and documented by MMSE and other neuropsychological exams. For our specific study, we added the inclusion criteria that the $\mathrm{AD}$ patients be nonsmokers, have no congenital bleeding abnormality and were not anticoagulated. After these inclusion criteria were met, whole blood $(5 \mathrm{ml})$ was obtained from a peripheral vein on one of the patients' arms. The blood sample was anticoagulated with sodium citrate ( 9 parts blood to 1 part $0.105 \mathrm{M}$ sodium citrate), centrifuged at $3000 \mathrm{xg}$ for $15 \mathrm{~min}$ at room temperature, with plasma removed, aliquoted and placed at $-80^{\circ} \mathrm{C}$ before transport to the University of Arizona. After all plasma samples were collected, they were sent on dry ice and kept at $-80^{\circ} \mathrm{C}$ prior to experimentation.

\section{Coagulation Analyses and Carboxyhemefibrinogen (COHF) Assay}

Plasma samples were thawed to $37^{\circ} \mathrm{C}$ just prior to analysis. Mixture volume for subsequently described samples was $359.4 \mu 1$. Samples were composed of $326 \mu 1$ of plasma; $10 \mu \mathrm{l}$ of tissue factor $(0.1 \%$ final concentration in $\mathrm{dH}_{2} \mathrm{O}$; Diagnostica Stago S.A.S., Asnieres sur Seine, France), $3.6 \mu \mathrm{l}$ of $\mathrm{dH}_{2} \mathrm{O}$ or CORM-2 (CO releasing molecule-2; tricarbonyldichlororuthenium (II) dimer, 100 $\mu \mathrm{M}$ final; Sigma-Aldrich, Saint Louis, MO, USA) and $20 \mu \mathrm{l}$ of $200 \mathrm{mM} \mathrm{CaCl}_{2}$ as previously noted in our COHF assay $[25,27,28]$. This amount of CORM-2 increases clot strength in a standardized manner when $\mathrm{CO}$ is not present in detectable concentrations $[25,27,28]$. Mixtures were pipetted into disposable plastic cups in a computercontrolled thrombelastograph ${ }^{\circledR}$ (Model 5000, Haemoscope Corp., Niles, IL, USA), with $\mathrm{CaCl}_{2}$ added to commence coagulation. Data were collected at $37^{\circ} \mathrm{C}$ for $15 \mathrm{~min}$. The elastic modulus-based parameters previously described [25, 27, 28] were determined, as detailed in the legend of Fig. (1) subsequently presented.

Utilizing this technique, hypercoagulability was defined as a total thrombus generation (TTG) value $>95 \%$ confidence interval value of the normal plasma data set. The presence of COHF was defined as the \% increase in TTG via CORM-2 exposure that was less than the average of such measurements in normal plasma samples. For this series the value used to define $\mathrm{COHF}$ presence was determined to be an increase in TTG of $<89 \%$.

\section{Iron Enhanced Coagulation Detection Assay}

Plasma mixtures were composed of $320 \mu 1$ of plasma, 20 $\mu 1 \mathrm{dH}_{2} \mathrm{O}$ or deferoxamine $(5.6 \mathrm{mM}$ final; Sigma-Aldrich) that were incubated at $37^{\circ} \mathrm{C}$ for $15 \mathrm{~min}$ in the thrombelastograph cups, before adding $20 \mu \mathrm{l}$ of $200 \mathrm{mM} \mathrm{CaCl}_{2}$ [27, 28]. Data were collected until the alpha value of the mixture was obtained, with comparison of the maximum rate of thrombus generation (MRTG) values being the indicator of iron mediated coagulation enhancement as previously noted [27, 28]. The nonspecific decrease in MRTG value secondary to deferoxamine addition was determined by the difference in the mean between ten replicates of two conditions (without and with deferoxamine) in pooled normal plasma (George King Bio-Medical) plus two standard deviations of the deferoxamine exposed replicates - this summated to a decrease of 1.2 dynes $/ \mathrm{cm}^{2} / \mathrm{sec}$. Thus, a decrease in MRTG $>1.2$ dynes $/ \mathrm{cm}^{2} / \mathrm{sec}$ after deferoxamine exposure would define the presence of iron mediated coagulation 

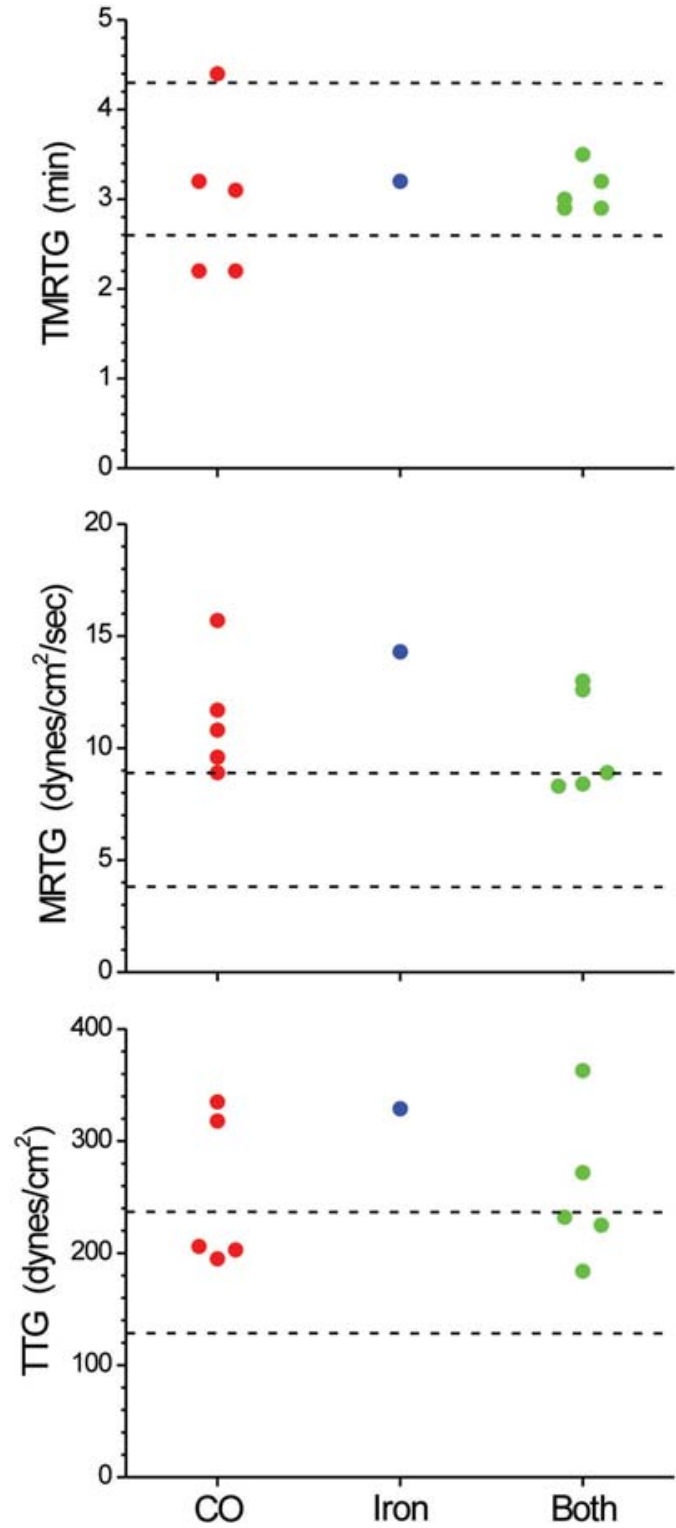

Fig. (1). Effect of Alzheimer's disease on TMRTG, MRTG and TTG stratified by modulation of coagulation by $\mathrm{CO}$, iron, or both. Time to maximum rate of thrombus generation (TMRTG): this is the time interval (min) observed prior to maximum speed of clot growth; maximum rate of thrombus generation (MRTG): this is the maximum velocity of clot growth observed (dynes/ $\mathrm{cm}^{2} / \mathrm{sec}$ ); and total thrombus generation (TTG, dynes $/ \mathrm{cm}^{2}$ ), the final viscoelastic resistance observed after clot formation. Individual data are represented by each circle. Dashed lines represent the $95 \%$ confidence interval values for coagulation derived from normal individuals as described in text. All patients had modification of coagulation by $\mathrm{CO}$, iron, or both; the primary kinetic abnormalities present were large rates of clot formation and greater than normal clot strength.

enhancement (upper limit of normal; lower limit of normal $<-0.8$ dynes $\left./ \mathrm{cm}^{2} / \mathrm{sec}\right)$.

\section{Fibrinolytic Kinetic Analyses}

Plasma samples were thawed to $37^{\circ} \mathrm{C}$ prior to analysis. The final mixture volume was $360 \mu \mathrm{l}$. The mixture was composed of $320 \mu \mathrm{l}$ of plasma; $10 \mu \mathrm{l}$ of tissue factor $(0.1 \%$
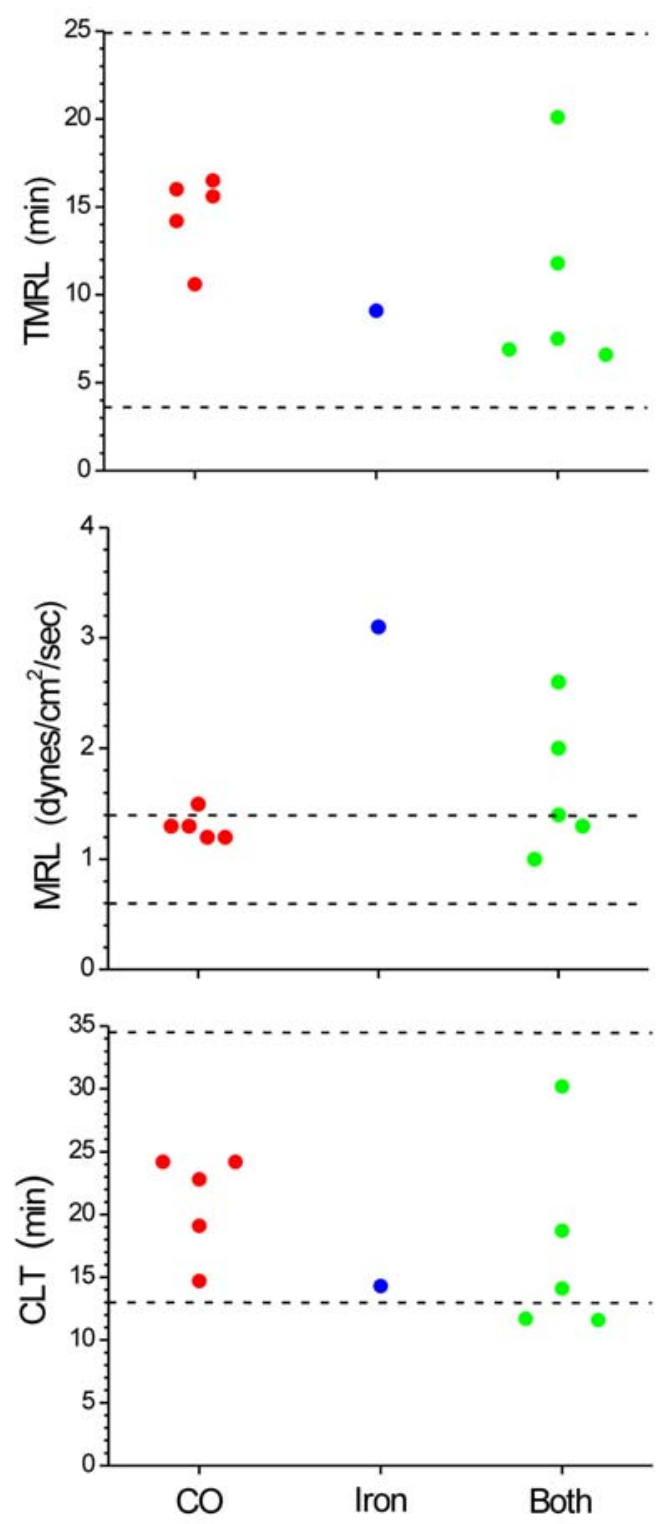

Fig. (2). Effect of Alzheimer's disease on TMRL, MRL and CLT stratified by modulation of fibrinolysis by $\mathrm{CO}$, iron, or both. Time to maximum rate of lysis (TMRL, min), maximum rate of lysis (MRL, dynes $/ \mathrm{cm}^{2} / \mathrm{sec}$ ) and clot lysis time (CLT, the time to reach 2 $\mathrm{mm}$ amplitude after maximum amplitude is achieved). Individual data are represented by each circle. Dashed lines represent the $95 \%$ confidence interval values for fibrinolysis derived from normal individuals as described in text. While most patients had a normal fibrinolytic kinetic profile, a few demonstrated enhanced vulnerability to clot lysis demonstrated by an abnormally increased rate of clot lysis and decreased clot lysis time.

final concentration in $\mathrm{dH}_{2} \mathrm{O}$; Diagnostica Stago S.A.S.), 10 $\mu l$ of tissue type plasminogen activator (tPA, $580 \mathrm{IU} / \mu \mathrm{g}$, Genentech, Inc., San Francisco, CA, USA; $100 \mathrm{IU} / \mathrm{ml}$ final), and $20 \mu \mathrm{l}$ of $200 \mathrm{mM} \mathrm{CaCl}_{2}$ [27, 28]. Mixtures were placed in a disposable plastic cup in a thrombelastograph ${ }^{\circledR}$ (Model 5000, Haemoscope Corp.), with $\mathrm{CaCl}_{2}$ added as the last step. Data were collected until clot lysis time (CLT) at $37^{\circ} \mathrm{C}$ for was observed. Elastic modulus-based parameters of fibrinolysis were documented as previously noted [28,28], with details outlined in the legend of Fig. (2). 
Table 1. Preparation methods for extensive fibrin fiber networks of healthy plasma with added $\mathrm{FeCl}_{3}$ and CORM-2.

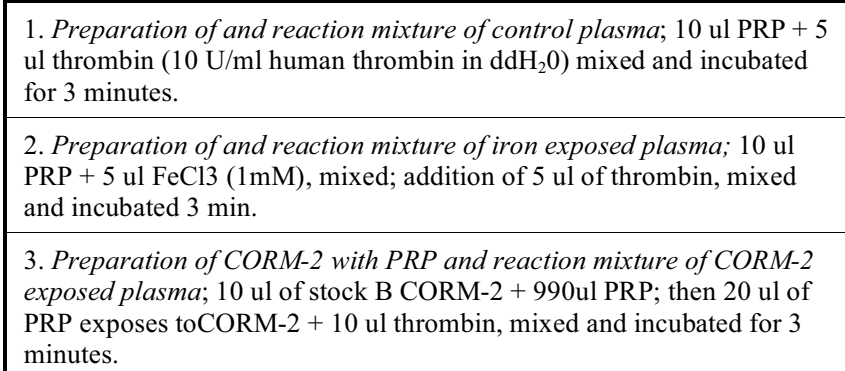

\section{SEM-Based Analyses}

All SEM-based studies were performed at the University of Pretoria. Ethical approval was granted by the Health Sciences Ethical committee of the University of Pretoria and written, informed consent was obtained from family members who act as guardians of the patients. Healthy individuals also filled in consent forms. Blood was obtained from three healthy, female subjects after written, informed consent was obtained. They were neversmokers, 25, 26 and 44 years old. Their plasma ferritin (range 2.6-3.3 g/L), serum ferritin (range 13-28 $\mathrm{ng} / \mathrm{ml}$ ) and iron saturation of ferritin (range 19-33\%) were normal. As noted previously, forty $\mathrm{ml}$ of whole blood was obtained, anticoagulated (sodium citrate, 9 parts blood to 1 part $0.105 \mathrm{M}$ sodium citrate), and platelet rich plasma (PRP) was generated by centrifugation of blood at $1250 \times \mathrm{xg}$ for $2 \mathrm{~min}[17,26]$.

Using a methodology previously noted to create extensive fibrin fiber networks, PRP smears with different compound exposures were made on glass cover slips and incubated at $37^{\circ} \mathrm{C}$ for 8 minutes. A $10 \mathrm{mM}$ CORM-2 stock solution was prepared by mixing $1 \mathrm{mg}$ CORM-2 and 195ul $100 \%$ DMSO, from here on referred to as stock A. Stock A was used to prepare a $1 \mathrm{mM}$ CORM-2 solution by adding $100 \mathrm{ul}$ of stock A to $900 \mathrm{ul}$ of phosphate buffered solution (PBS), form hereon referred to as stock B. Stock B was used in the following in the ratios shown in Table $\mathbf{1}$ and incubated with PRP for 5 minutes. PRP smears after exposure to different compounds (see Table 1) were made on glass coverslips.

The cover slips were placed in $0.075 \mathrm{M}$ PBS on a shaker and washed for 20 minutes. The samples were then fixated for 30 minutes followed by three washing steps in $0.075 \mathrm{M}$ PBS for three minutes to remove any residual fixative. The smears were then post-fixated for 15 minutes with $1 \%$ osmium tetroxide $\left(\mathrm{OsO}_{4}\right)$, followed by a washing process, for three minutes in $0.075 \mathrm{M}$ PBS. The samples were finally dehydrated serially in $30 \%, 50 \%, 70 \%, 90 \%$ and then three times in $100 \%$ ethanol followed by drying using hexamethyldisilazane; mounting and coating with carbon. Once the samples had been coated they were analyzed with a scanning electron microscope (Zeiss ULTRA plus FEG SEM, Carl Zeiss Microscopy GmbH, Jena, Germany).

Blood was obtained from AD patients $(n=12)$ who were diagnosed by qualified medical practitioners. All AD individuals were diagnosed using the MMSE. The AD patients were divided into 2 groups (normal and high serum ferritin concentrations, high ferritin being defined as a level of $>120 \mathrm{ng} / \mathrm{ml}$ for females and $>250 \mathrm{ng} / \mathrm{ml}$ for males). The AD patients did not have any chronic medical conditions, were nonsmokers, and the age of these patients was $78 \pm 12$ $($ mean $\pm \mathrm{SD})$ years. Fibrin fiber networks were generated as per the same methods described for healthy individuals, but without addition of iron or CORM-2.

After visualization of the fibrin networks with SEM, micrographs were obtained at 40,000 times machine magnification. A representative micrograph of each sample was selected, and subsequently a 10x5 grid was superimposed onto the picture. One fiber was randomly selected out of every block of the grid, ensuring that fiber measurements were not duplicated, and that fibers were systematically assessed to prevent observer bias [29, 30]. Fibrin fiber thickness was measured with ImageJ (ImageJ is a public domain, Java-based image processing program developed at the National Institutes of Health: http://rsbweb.nih.gov/ij/). From each sample, 50 fibers were measured.

\section{Statistical Analyses and Graphics}

Data are presented as mean $\pm \mathrm{SD}$, individual parameter values, $\%$ incidence with $95 \%$ confidence intervals $(\mathrm{CI})$, or simply $95 \%$ CI. Coagulation and fibrinolysis parameters of AD patient samples were compared with $95 \%$ confidence intervals generated from normal plasma. A statistical program was used for student's t-test analyses of differences in fibrin polymer fiber widths (SigmaStat 3.1, Systat Software, Inc., San Jose, CA, USA). Graphics depicting viscoelastic data were generated with a commercially available program (OrigenPro 7.5, OrigenLab Corporation, Northampton, MA, USA; CorelDRAW12, Corel Corporation, Mountain View, CA, USA).

\section{RESULTS}

\section{Viscoelastic Analyses}

Eleven AD patients ( 7 male), aged $73 \pm 10$ years, with MMSE values of $20 \pm 3$ and body mass index (BMI) values of $24 \pm 3 \mathrm{~kg} / \mathrm{m}^{2}$ were recruited. In regard to comorbidities, no patient had diabetes mellitus, one had a history of migraine, one had a history of myocardial infarction, four had hypertension, and one had a history of asthma. We had originally requested 10 patients to study, but an additional patient had blood collected, so the vendor was kind enough to donate the plasma and clinical information of this patient for our study.

As for normal plasma values, the $95 \%$ confidence interval values for coagulation and fibrinolytic parameters are depicted in Table 2. For an AD patient to be considered hyper/hypocoagulable or hyper/hypofibrinolytic, the kinetic parameter value of interest had to be outside the normal $95 \%$ $\mathrm{CI}$ values. The criteria for COHF formation and iron enhancement of coagulation was presented in the materials and methods section previously.

Fig. (1) displays the coagulation profiles of the $A D$ patients stratified by COHF formation (CO enhancement of coagulation), enhancement of coagulation by iron, or 
Table 2. Normal $95 \%$ CI values for coagulation and fibrinolytic kinetic parameters.

\begin{tabular}{|c|c|}
\hline Parameter & Values \\
\hline \hline TMRTG $(\mathrm{min})$ & $2.6-4.3$ \\
\hline MRTG $\left(\right.$ dynes $\left./ \mathrm{cm}^{2} / \mathrm{sec}\right)$ & $3.8-8.9$ \\
\hline TTG $\left(\right.$ dynes $\left./ \mathrm{cm}^{2}\right)$ & $127-237$ \\
\hline TMRL $(\mathrm{min})$ & $3.6-24.9$ \\
\hline MRL $\left(\right.$ dynes $\left./ \mathrm{cm}^{2} / \mathrm{sec}\right)$ & $0.6-1.4$ \\
\hline CLT $(\mathrm{min})$ & $13.0-34.5$ \\
\hline
\end{tabular}

Time to maximum rate of thrombus generation (TMRTG): this is the time interval (min) observed prior to maximum speed of clot growth; Maximum rate of thrombus generation (MRTG): this is the maximum velocity of clot growth observed (dynes/cm2/sec); Total thrombus generation (TTG): this is the total area under the velocity curve during clot growth (dynes/cm2), representing the amount of clot strength generated during clot growth; time to maximum rate of lysis (TMRL): defined as the time when maximum amplitude is observed until the time (min) of maximum velocity of clot lysis is observed; maximum rate of lysis (MRL): the greatest velocity of clot lysis (dynes/cm2/sec); CLT: defined as the time (min) from when growth ceases until lysis is complete (amplitude returns to $2 \mathrm{~mm}$ ).

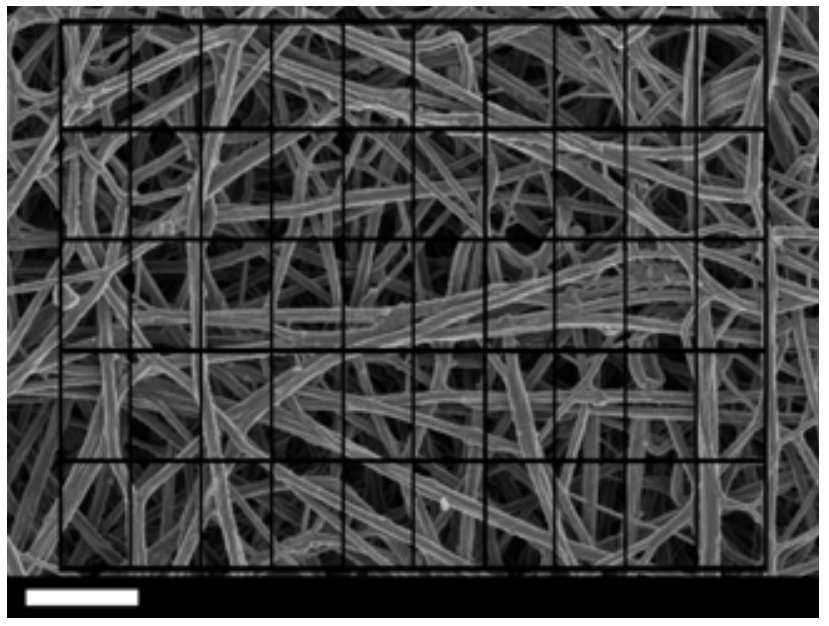

Fig. (3). Healthy fibrin fiber network. Plasma with thrombin added to create an extensive fibrin network is depicted. Ten ul of plasma was added to $5 \mathrm{ul}$ thrombin $(10 \mathrm{U} / \mathrm{ml}$ human thrombin in ddH20), mixed and incubated for 3 minutes to create an extensive fibrin network. Individual fibrin fibers are visible. A typical 5 by 10 grid is superimposed, which was used to assess fibrin fiber diameter as discussed in text. Scale bar $=1 \mu \mathrm{m}$.

enhancement of coagulation by both $\mathrm{CO}$ and iron. Of interest, there was no patient without modulation of coagulation by either $\mathrm{CO}$ or iron. With regard to $\mathrm{CO}$, ten of eleven patients had COHF formation, whereas seven patients had iron enhanced coagulation kinetics. With regards to TMRTG, one patient had a hypocoagulable TMRTG value, two patients had hypercoagulable TMRTG values, with the remainder within the normal range. As for MRTG, seven patients had hypercoagulable values, two patients had values exactly at the $95 \%$ CI value of normal, with the balance within normal range. In the case of TTG, five patients had abnormally strong thrombi, with the balance being within the normal range.
Fig. (2) displays the fibrinolysis profile data of $\mathrm{AD}$ patients stratified as seen in Fig. (1). With regard to onset of clot lysis, all AD patients had normal TMRL values. However, four AD patients had an above normal increase in MRL values, indicating an increased vulnerability to lysis, while the remainder of the group had values in the normal range. Lastly, only two of the eleven AD patients had CLT values that were below normal values, indicative of a hyperfibrinolytic tendency in these individuals.

\section{SEM-Based Analyses}

The AD cohort from the University of Pretoria was composed of ten women and two men; the normal ferritin group had a serum ferritin concentration of $66 \pm 33 \mathrm{ng} / \mathrm{ml}$ whereas the high ferritin group had a significantly $(\mathrm{P}=0.0003)$ ferritin concentration of $253 \pm 88 \mathrm{ng} / \mathrm{ml}$.

With regard to SEM data, Fig. (3) shows a typical, normal subject fibrin network with mostly major, thick fibrin fibers. In healthy individuals, individual fibrin fibers are visible, appearing like a net, with no fused fibers and no fine, lattice-like appearance. When $\mathrm{FeCl}_{3}$ is added to PRP followed by exposure to thrombin, (Fig. 4A) a finer fiber lattice net is observed (see thick white arrow), as well as areas forming a denser mesh $\left(\right.$ see $\left.^{*}\right)$, where the lattice net fuses to form a matted deposit. Close examination revealed areas of denser fibrin deposits $(*)$, consisting of fine fibers (see thin white arrow) that were packed closely to each other.

Addition of CORM-2 to PRP, followed by exposure to thrombin, resulted in fibrin fiber diameters that were also decreased compared to the healthy fibers but were more densely packed, with a more globular structure compared to the rather straight individual fibers seen in the healthy fibrin structure (Fig. 4B). This ultrastructure was suggestive of CORM-2 modifying the fibrin packaging. Fig. (5A and $\mathbf{B}$ ) compared high magnification healthy fibrin fibers to that of healthy PRP exposed to CORM-2.

Fig. (6A-F) shows fibrin networks from the individual AD patients with normal serum ferritin levels. Here we see that the fiber diameter increases. Fig. (7A-F) shows fibrin networks of Alzheimer's patients with high serum ferritin levels. The fibrin fiber nets resemble that seen in Fig. (4A) ( $\mathrm{FeCl}_{3}$ is added to PRP followed by thrombin exposure), where the net resembles a fine lattice. We also noted that the individual fibrin fibers of both the normal and high serum ferritin AD individuals showed a more globular morphology, as seen with CORM-2, instead of the rather straight and smooth individual fibers seen in the healthy subject fibrin fibers. See arrows indicating globular structure in Fig. (6 and 7).

With regard to average fibrin fiber width, the value for the normal subjects was $105 \pm 3 \mathrm{~nm}$. Exposure to $\mathrm{FeCl}_{3}$ decreased fiber width by $58 \%$ and exposure to CORM-2 decreased width by $43 \%$. The average width values for the AD patients, stratified by serum ferritin concentration, are depicted in Fig. (8). The high ferritin AD group had a fiber width that was significantly less than the normal ferritin AD group, with an average value 34\% less than the high ferritin group. 


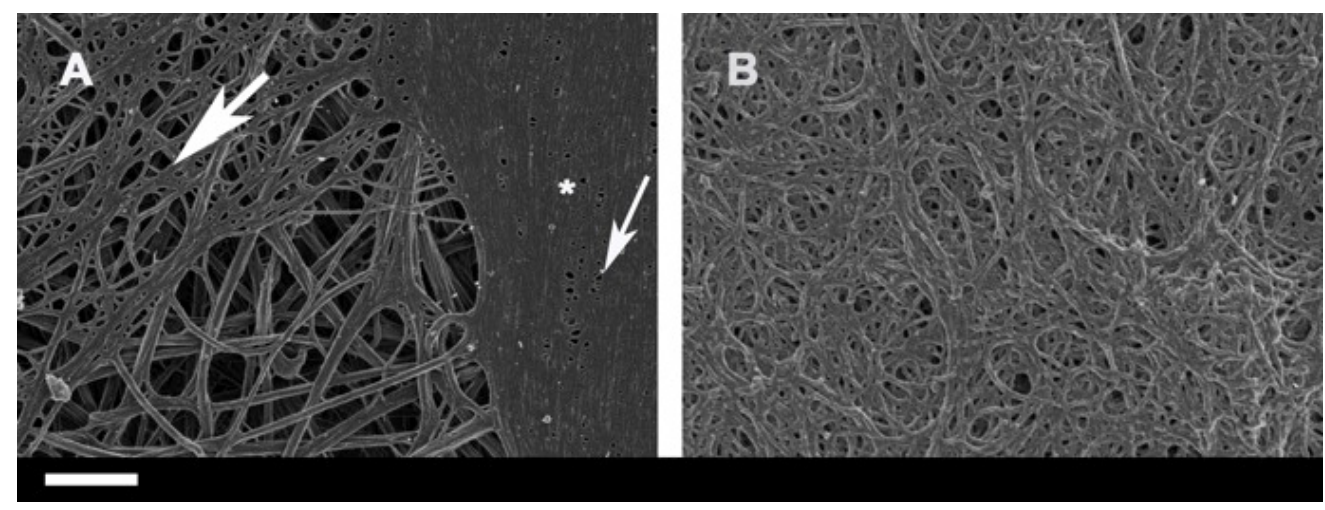

Fig. (4). Healthy fibrin fiber network with added $\mathrm{FeCl}_{3}$ (A) and CORM-2 (B) and thrombin to create an extensive fibrin network. (A) 10 ul PRP was added to $5 \mathrm{ul} \mathrm{FeCl}_{3}(1 \mathrm{mM})$ and mixed followed by addition of $5 \mathrm{ul}$ of thrombin; this was mixed again and incubated 3 min. Thick arrow (left) -fine fiber lattice net; * - areas of denser fibrin deposits, consisting of fine fibers (see thin arrow (right)) that are only packed closer to each other. (B) $10 \mathrm{ul}$ of stock B CORM-2 was added to 990ul PRP (see table 1 for CORM-2 preparation); then 20 ul of PRP was exposed to CORM-2 followed by addition of $10 \mathrm{ul}$ thrombin; this was mixed and incubated for 3 minutes. Globular fibrin packaging is visible, without typical straight fibrin fibers seen in healthy fibrin nets. Scale bar $=1 \mu \mathrm{m}$.

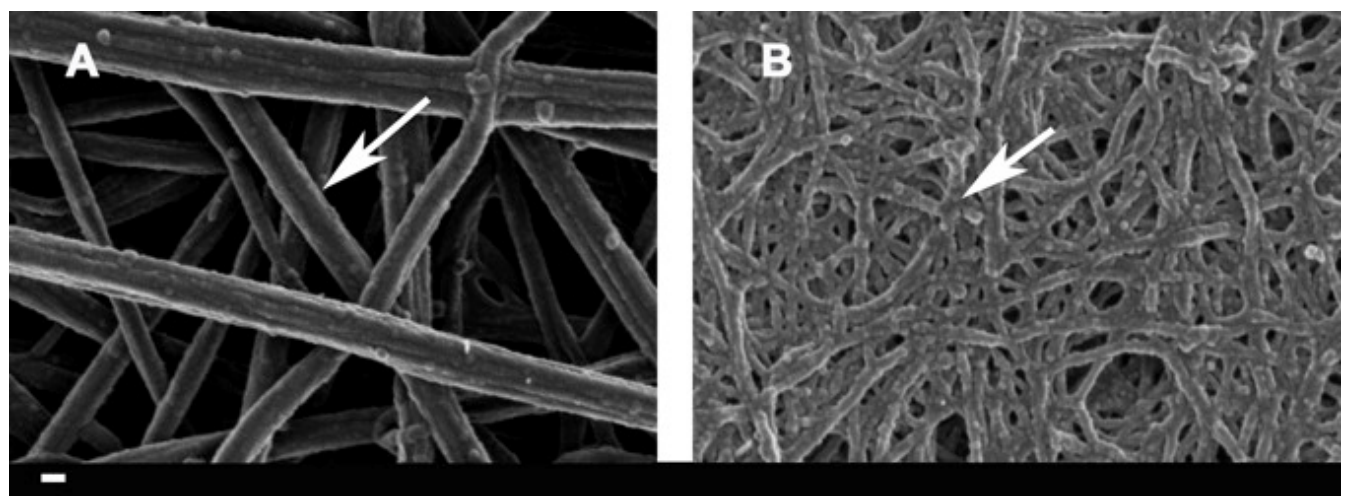

Fig. (5). High machine magnification (100,000x machine magnification) of healthy fibrin fiber network (A) and with added CORM-2 (B)_and thrombin to create an extensive fibrin network. Preparation as described in figure 4. Arrows show smooth and straight fibrin fiber packaging in (A) and globular fibrin fiber structure in (B). Scale bar $=100 \mathrm{~nm}$.
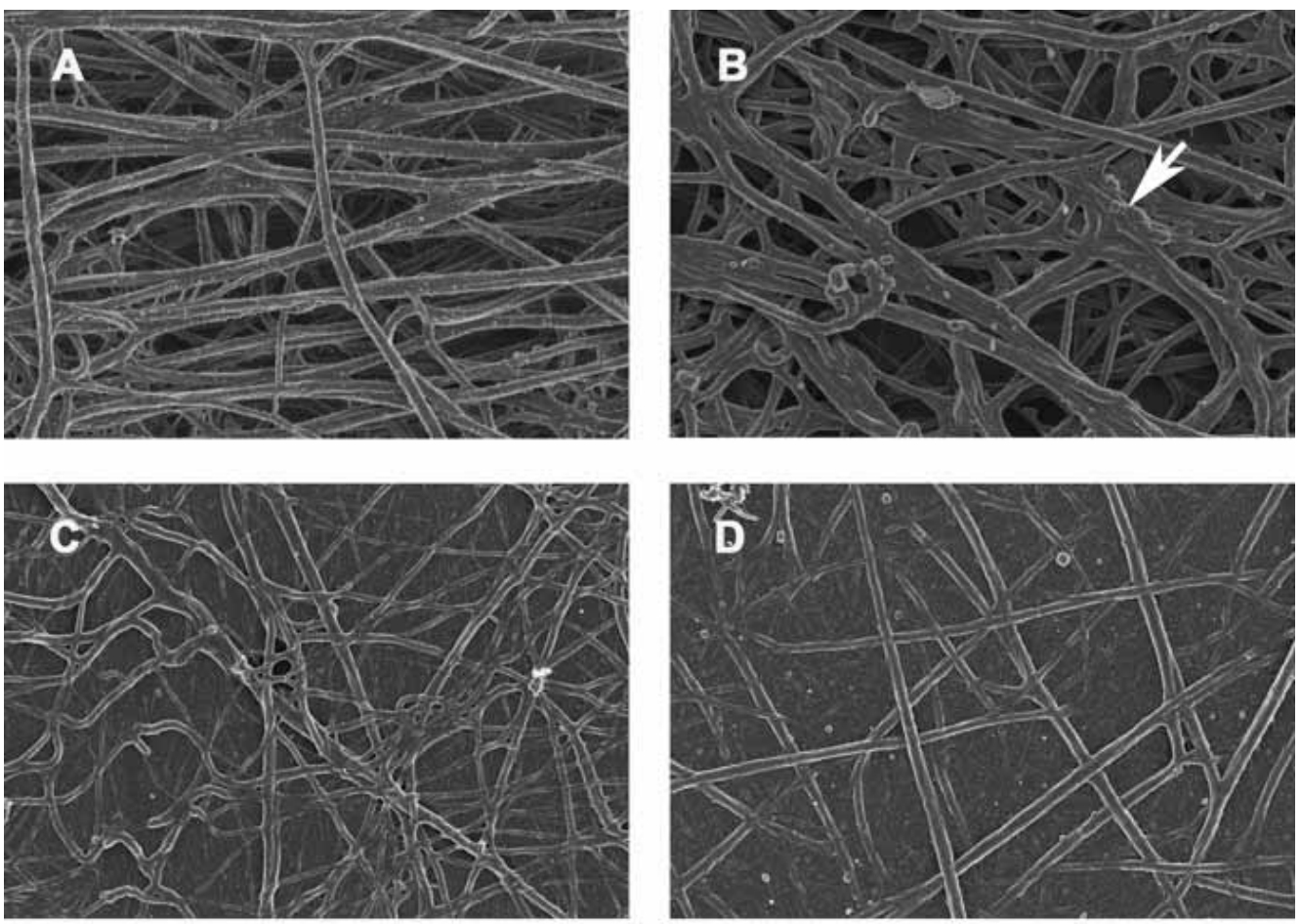

Fig. (6). Contd... 


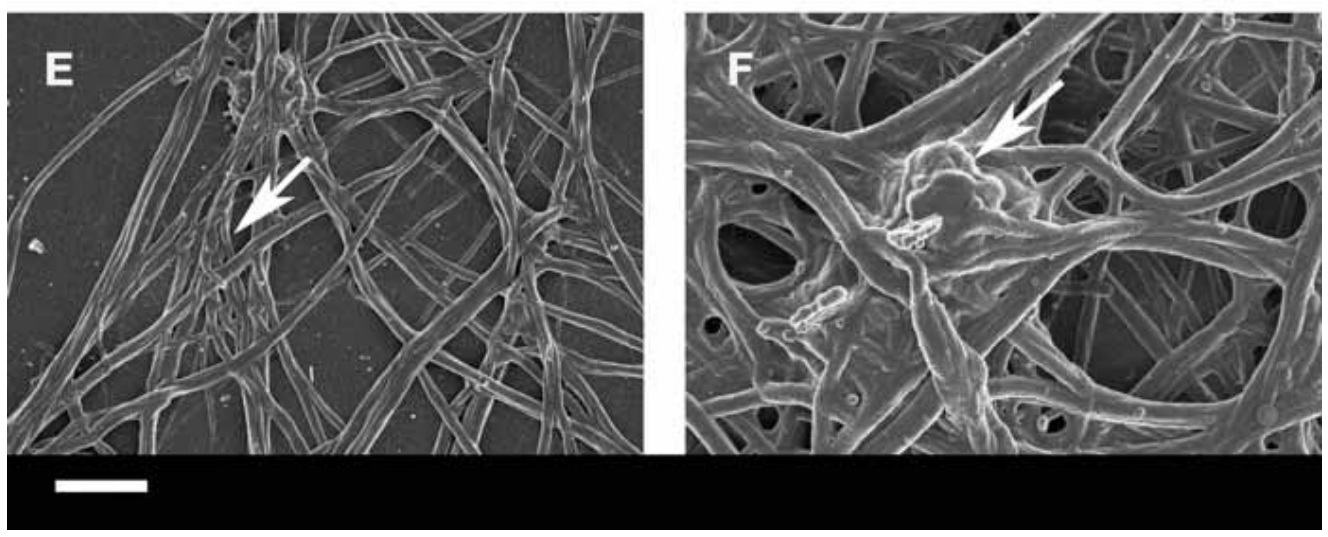

Fig. (6). Fibrin fiber networks of six AD patients (A-F) with normal serum ferritin levels. Ten ul of AD plasma was added to 5 ul thrombin $\left(10 \mathrm{U} / \mathrm{ml}\right.$ human thrombin in $\left.\mathrm{ddH}_{2} 0\right)$, mixed and incubated for 3 minutes to create an extensive fibrin network. Healthy values for ferritin are 20 to $250 \mathrm{ng} / \mathrm{ml}$ for males and 10 to $120 \mathrm{ng} / \mathrm{mL}$ for females. Globular areas, similar to that observed with CORM-2 exposure are indicated with arrows. Thus, more of a CO effect on fibrin polymer formation is likely. Scale bar $=1 \mu \mathrm{m}$.
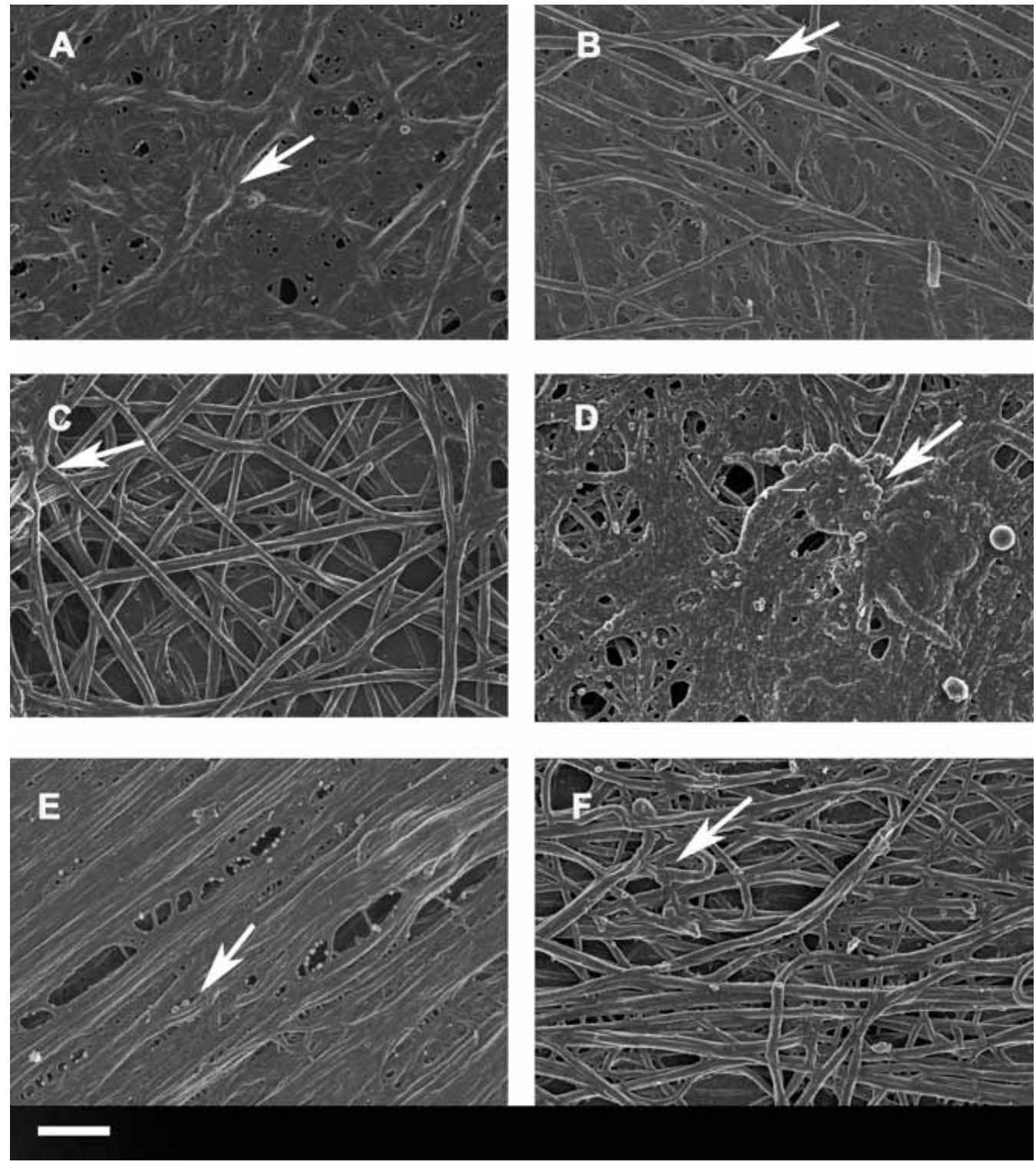

Fig. (7). Fibrin fiber networks of six AD patients (A-F) with high serum ferritin levels. Ten ul of AD plasma was added to 5 ul thrombin (10 $\mathrm{U} / \mathrm{ml}$ human thrombin in $\mathrm{ddH}_{2} 0$ ), mixed and incubated for 3 minutes to create an extensive fibrin network. Globular areas, similar to that observed with CORM-2 exposure are indicated with arrows. Unlike patients' clots in figure 6, both fine fiber lattice and globular areas are present, consistent with a combination of iron and CO mediated changes in fibrin polymer formation. Scale bar $=1 \mu \mathrm{m}$. 


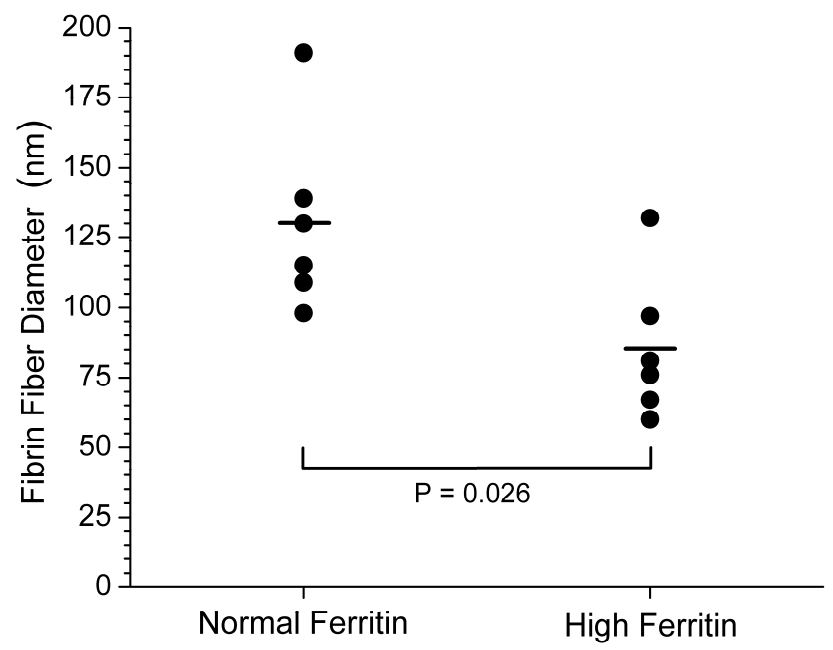

Fig. (8). Comparison of fibrin fiber diameter in $\mathrm{AD}$ patients with normal or high serum ferritin concentrations. Individual patient data is depicted as black dots; the small horizontal bars are the group mean values. AD patients with high serum ferritin concentrations had significantly smaller diameter fibrin strands compared to those with normal ferritin concentrations as indicated by the large horizontal bar. This difference is consistent with iron modification of fibrin polymer formation.

\section{DISCUSSION}

The most prominent observations of the present investigation include the finding that all patients afflicted with AD evaluated with viscoelastic methods had modulation of plasmatic coagulation by $\mathrm{CO}$, iron, or both. Of equal and critical importance, the ultrastructure of plasma clots obtained from AD patients displayed morphological features consistent with both iron and $\mathrm{CO}$ exposure, similar to normal plasma exposed to $\mathrm{FeCl}_{3}$ and CORM-2. We further stratified the $\mathrm{AD}$ patients by plasma ferritin concentration, not just for being at risk of exposure to greater circulating iron concentrations but also as an indirect marker of increased Hmox activity as has been demonstrated previously $[31,32]$. Thus, the significantly smaller fibrin fiber diameter observed in $\mathrm{AD}$ patients with high serum ferritin levels very well may be secondary to increased iron and $\mathrm{CO}$ secondary to more pronounced cerebral Hmox activity (Fig. 8). In sum, the coagulation kinetic and ultrastructural data support the concept of exposure of the circulation to increased concentrations of iron and $\mathrm{CO}$, and given the relative absence of confounding comorbidity in both cohorts of $\mathrm{AD}$ patients, the most likely source of iron and $\mathrm{CO}$ was $\mathrm{AD}$ associated, increased cerebral Hmox activity.

In contrast to the aforementioned coagulation and ultrastructural data, the observation that most of the $\mathrm{AD}$ patients undergoing viscoelastic analyses had normal to increased vulnerability to tPA mediated lysis was an unexpected finding (Fig. 2). Indeed, four patients had abnormally great rates of clot lysis and two had abnormally decreased clot lysis times. It would be expected that increased $\mathrm{CO}$ and iron would attenuated fibrinolysis in the absence of any other biochemical aberration [17]. Thus, the data of the present investigation support the concept that an endogenous fibrinolysin may have been present despite increased Hmox activity, as has been documented by us in the settings of obesity and cancer $[33,34]$.

When our data are considered as a whole, $\mathrm{CO}$ and iron enhanced coagulation could play a role in spontaneous cerebral embolus formation in the setting of $\mathrm{AD}$ as documented by previous ultrasonic investigations [11, 12]. Further, when coupled with normal or accelerated fibrinolysis, it is possible that microembolic or in situ thrombus formation within the brain occurs causing ischemic damage, but with timely lysis preventing large, clinically detectable neurologic defects. Nevertheless, over a period of years, it is possible that such a pathological state of coupled microischemic stroke/fibrinolysis could eventually result in minimal cognitive impairment and ultimately $\mathrm{AD}$. The notion that inhibition of Hmox chronically could perhaps greatly delay the onset of $\mathrm{AD}$ is borne out in experiments with transgenic mice that mimic $\mathrm{AD}$, as both behavioral and neuroanatomical changes that occurred over time were prevented with Hmox inhibition [24]. In sum, only human studies using this therapeutic approach, perhaps at first in those with minimal cognitive defect, will help define the part played by Hmox and the specific mechanism(s) by which Hmox contributes to AD.

While promising, the data of the present investigation serve primarily as a "proof-of-concept" of the involvement of $\mathrm{CO}$ and iron interactions with the circulation as a possible contributor to $\mathrm{AD}$. Utilizing our analytical methods to correlate $\mathrm{CO}$ and iron mediated modulation of coagulation and plasmatic hypercoagulability with clinical progression of dementia in critical long term studies remains to be performed. If increased cerebral Hmox activity plays a role in the progression of dementia [18-21], then our hematological methodology might be utilized to correlate changes in coagulation with changes in brain pathology (e.g., tissue iron deposition). Thus, if novel therapeutic innovations (e.g., Hmox inhibition, iron chelation) are tested clinically to slow or abrogate dementia, the efficacy of such interventions can be assessed with simultaneous clinical assessment, magnetic resonance imaging, and viscoelastic/ultrastructural analyses. At a minimum, we plan to establish such correlations with progression of disease in future investigations.

In conclusion, our study demonstrated that two small cohorts of AD patients had viscoelastic and ultrastructural evidence of $\mathrm{CO}$ and iron modulation of coagulation. Further, hypercoagulability was observed, with velocity of clot growth the most prominent feature - and iron and $\mathrm{CO}$ both increase clot growth velocity [17]. This enhancement of coagulation was coupled with normal to enhanced vulnerability to lysis, perhaps significantly contributing to ephemeral microembolic phenomena that only over time result in clinical dementia. Future study of the part played by Hmox-generated $\mathrm{CO}$ and iron in the pathogenesis of dementia and concurrent hypercoagulability is warranted.

\section{CONFLICT OF INTEREST}

The authors confirm that this article content has no conflict of interest. 


\section{ACKNOWLEDGEMENTS}

This investigation was supported by the Departments of Anesthesiology and Physiology.

\section{REFERENCES}

[1] Armstrong RA. A critical analysis of the 'amyloid cascade hypothesis'. Folia Neuropathol 2014; 52(3): 211-5.

[2] Karim S, Mirza Z, Kamal MA, et al. The role of viruses in neurodegenerative and neurobehavioral diseases. CNS Neurol Disord Drug Targets 2014; 13(7): 1213-23.

[3] Stone J, Johnstone DM, Mitrofanis J, O'Rourke M. The mechanical cause of age-related dementia (Alheimer's Disease): the brain is destroyed by the pulse. J Alzheimers Dis 2014, in press, PMID: 25318547.

[4] Gonzalez-Dominguez R, Garcia-Barrera T, Gomez-Ariza J. Homeostasis of metals in the progression of Alzheimer's disease. Biometals 2014; 27(3): 539-49.

[5] Crespo AC, Silva B, Marques L, et al. Genetic and biochemical markers in patients with Alzheimer's disease support a concerted systemic homeostasis dysregulation. Neurobiol Aging 2014; 35(4): 777-85.

[6] Mitra J, Guerrero EN, Hegde PM, et al. New perspectives on oxidized genome damage and repair inhibition by pro-oxidant metals in neurological diseases. Biomolecules 2014; 4(3): 678-703.

[7] Braidy N, Poljak A, Marjo C, et al. Metal and complementary molecular bioimaging in Alzheimer's disease. Front Aging Neurosci 2014; 6:138

[8] Grunblatt E, Bartl J, Riederer P. The link between iron, metabolic syndrome, and Alzheimer's disease. J Neural Transm 2011; 118(3): 371-9.

[9] Letra L, Santana I, Seica R. Obesity as a risk factor for Alzheimer's disease: the role of adipocytokines. Metab Brain Dis 2014; 29(3): 563-8.

[10] Rios JA, Cisternas P, Arrese M, Barja S, Inestrosa NC. Is Alzheimer's disease related to metabolic syndrome? A Wnt signaling conundrum. Prog Neurobiol 2014; 121C: 125-46.

[11] Purandare N, Burns A, Daly KJ, et al. Cerebral emboli as a potential cause of Alzheimer's disease and vascular dementia: casecontrol study. BMJ 2006; 332(7550): 1119-24.

[12] Purandare N, Voshaar RC, Morris J, et al. Asymptomatic spontaneous cerebral emboli predict cognitive and functional decline in dementia. Biol Psychiatry 2007; 62(4): 339-44.

[13] Gupta A, Watkins A, Thomas P, et al. Coagulation and inflammatory markers in Alzheimer's and vascular dementia. Int J Clin Pract 2005; 59(1): 52-7.

[14] Xu G, Zhang H, Zhang S, Liu X. Plasma fibrinogen is associated with cognitive decline and risk for dementia in patients with mild cognitive impairment. Int J Clin Pract 2007; 62(7): 1070-5.

[15] Lipinski B, Pretorius E. The role of iron-induced fibrin in the pathogenesis of Alzheimer's disease and the protective role of magnesium. Front Hum Neurosci 2013; 7: 735.

[16] Bester J, Buys AV, Lipinski B, Kell DB, Pretorius E. High ferritin levels have major effects on the morphology of erythrocytes in Alzheimer's disease. Front Aging Neurosci . 2013; 5: 88.

[17] Nielsen VG, Pretorius E. Iron and carbon monoxide enhance coagulation and attenuate fibrinolysis by different mechanisms. Blood Coagul Fibrinolysis 2014; 25(7): 695-702.
[18] Barone E, Di Domenico F, Mancuso C, Butterfield DA. The Janus face of the heme oxygenase/biliverdin reductase system in Alzhiemer disease: It's time for reconciliation. Neurobiol Dis 2014; 62: 144-59.

[19] Barone E, Di Domenico F, Sultana R, et al. Heme oxygenase-1 posttranslational modifications in the brain of subjects with Alzhiemer disease and mild cognitive impairment. Free Radic Biol Med 2012; 52(11-12): 2292-301.

[20] Di Domenico F, Barone E, Mancuso C, et al. HO-1/BVR-A system analysis in plasma from probable Alzheimer's disease and mild cognitive impairment subjects: a potential biochemical marker for the prediction of the disease. J Alzheimers Dis 2012; 32(2): 277-89.

[21] Song W, Zukor H, Liberman A, et al. Astroglial heme oxygenase-1 and the origin of corpora amylacea in aging and degenerating neural tissues. Exp Neurol 2014; 254: 78-89.

[22] Leffler CW, Parfenova H, Jaggar JH. Carbon monoxide as an endogenous vascular modulator. Am J Physiol Heart Circ Physiol 2011; 301(1): H1-H11.

[23] Queiroga CS, Vercelli A, Vieira HL. Carbon monoxide and the CNS: challenges and achievements. Br J Pharmacol 2014; in press, doi:10.1111/bph.12729.

[24] Gupta A, Lacoste B, Pistel PJ, et al. Neurotherapeutic effects of novel HO-1 inhibitors in vitro and in a transgenic mouse model of Alzheimer's disease. J Neurochem 2014; 131(6): 778-90.

[25] Nielsen VG, Arkebauer MR, Vosseller K. Redox-based thrombelastographic method to detect carboxyhemefibrinogen mediated hypercoagulability. Blood Coagul Fibrinolysis 2011; 22(8): 657-61.

[26] Nielsen VG, Pretorius E. Iron enhanced coagulation is attenuated by chelation: a thrombelastographic and ultrastructural analysis. Blood Coagul Fibrinolysis, 2014; 25(8): 845-50.

[27] Matika RW, Nielsen VG, Steinbrenner EB, Sussman AN, Madhrira M. Hemodialysis patients have plasmatic hypercoagulability and decreased fibrinolytic vulnerability: role of carbon monoxide. ASAIO J 2014; 60(6): 716-21.

[28] Thompson III JL, Nielsen VG, Castro A, Chen A. Heme oxygenase derived carbon monoxide and iron mediated plasmatic hypercoagulability in a patient with calcific mitral valve disease. $\mathrm{J}$ Thromb Thrombolysis 2014, in press, DOI 10.1007/s11239-0141134-x.

[29] Pretorius E. Quantifying changes in fibrin fiber network morphology. Ultrastruct Pathol 2011; 35(4): 150-4.

[30] Pretorius E, Steyn H, Engelbrecht M, Swanepoel AC, Oberholzer HM. Differences in fibrin fiber diameters in healthy individuals and thromboembolic ischemic stroke patients. Blood Coagul Fibrinolysis 2011; 22(8): 696-700.

[31] Kirino Y, Takeno M, Iwasaki M, et al. Increased serum HO-1 in hemophagocytic syndrome and adult-onset Still's disease: use in the differential diagnosis of hyperferritinemia. Arthritis Res Ther 2005;7(3): R616-24.

[32] Le Blanc S, Villarroel P, Candia V, et al. Type 2 diabetic patients and their offspring show altered parameters of iron status, oxidative stress and genes related to mitochondrial activity. Biometals 2012; 25(4): 725-35

[33] Nielsen VG, Galvani CA, Boyle PK, Steinbrenner EB, Matika RW. Bariatric patients have plasmatic hypercoagulability and systemic upregulation of hemeoxygenase-1 activity. Blood Coagul Fibrinolysis 2015, in press, PMID: 25101516.

[34] Nielsen VG, Matika RW, Ley MLB, et al. Tissue type plasminogen activator induced fibrinolysis is enhanced in patients with breast, lung, pancreas and colon cancer. Blood Coagul Fibrinolysis 2014; 25(3): 248-53. 